Vacuum Vol. 46, $\mathrm{n}^{\circ} 12$, p. 1385 (1995))

\title{
Microcrystalline silicon thin films prepared by RF reactive magnetron sputter deposition
}

\author{
M F Cerqueira, M Andritschky, L Rebouta, J A Ferreira and M.F da Silva*, \\ Departamento de Física, Universidade do Minho, Campus de Gualtar, Braga, 4710-057 Portugal \\ "INETI/ICEN, Departamento de Fisica, E.N.10, P-2685 Sacavém, Portugal
}

\begin{abstract}
Hydrogenated microcrystalline silicon $(\mu \mathrm{c}-\mathrm{Si}: \mathrm{H})$ thin films with $\mathrm{Cu}$ as a dopant material (about 2 wt.\%) were deposited by RF planar magnetron sputtering in an argon/hydrogen plasma. The composition and microstructure of the films were analysed by SEM, ERD/RBS, X-ray diffraction and Raman spectroscopy.

These techniques revealed a columnar film structure, each column consisting of several small (nano) crystals with a lateral dimension up to $10 \mathrm{~nm}$. The crystals are oriented, generally with the (111) plane parallel to the sample surface. The hydrogen content of the thin films is about 27-33 at.\%. Low deposition rates and low sputter gas pressures favour crystallisation and grain growth. The behaviour can be understood in terms of the diffusion or relaxation length $\Lambda$ of the deposited Si-atoms.
\end{abstract}

\section{Introduction}

Hydrogenated microcrystalline silicon $(\mu \mathrm{c}-\mathrm{Si}: \mathrm{H})$ has attracted considerable attention as a potential material for electronic devices such as solar cells, thin film transistors and MOSFETs. By close control of the deposition technique it is possible to tailor the electrical and optical properties of $\mu \mathrm{c}-\mathrm{Si}: \mathrm{H}$ thin films. The thin film microstructure, which is defined by the ratio of the amorphous and crystalline $\mathrm{Si}$ components, the geometry of the crystalline phase, the film density and hydrogen content and type of $\mathrm{Si}-\mathrm{H}$ bonding, will determine the above mentioned electronic characteristics and long term stability of any device containing $\mu \mathrm{c}-\mathrm{Si}: \mathrm{H}$ thin films ${ }^{1}$. These films are generally described as consisting of spherical crystals embedded in an amorphous $\mathrm{Si}-\mathrm{H}$ matrix. The grain size ranges typically from $3 \mathrm{~nm}$ to $100 \mathrm{~nm}$, whereby the lower limit is defined by thermodynamical considerations. The films can be deposited by high and low temperature deposition processes such as chemical vapour deposition, glow discharge techniques or plasma reactor ${ }^{2-5}$. Another technique is the sputter deposition process, which is a commercially interesting technique due to large area deposition at reasonable deposition rates avoiding hazardous gas handling and also allowing doping by simple means. Furthermore, the sputter deposition technique is able to control the structural thin film properties within a wide range. 
Nevertheless, it should be mentioned that physical vapour deposition produces thin films which may be in a non-equilibrium state and may therefore contain crystals smaller than $3 \mathrm{~nm}$.

In this work, we prepared $\mu \mathrm{c}-\mathrm{Si}: \mathrm{H}$ thin films by planar RF magnetron sputtering of $\mathrm{Si}$ in a hydrogen (and argon) atmosphere at low pressure. The physical properties of the thin films were analysed by Raman spectroscopy, Scanning Electron Microscopy (SEM), X-ray diffraction, Elastic Recoil Detection (ERD) and Rutherford Backscattering Spectroscopy (RBS) measurements. In the first part of the work we will describe the microstructure of the thin films produced. In the second part we will try to relate phenomenally the microstructure of the thin films to the intrinsic parameters of the sputter process using the diffusion length $\Lambda$ of condensed $\mathrm{Si}$-atoms as a single parameter. Details of electrical and optical thin film properties and of the nature of the existing hydrogen bonds will be published elsewhere.

\section{Experimental}

Although we used silicon substrates for some measurements (FTIR), most results reported in this article are based on thin films of $\mu \mathrm{c}-\mathrm{Si}: \mathrm{H}$ on microscopy glass slides. These substrates were introduced into an Alcatel SCM 650 sputter machine and the radio frequency RF (13.56 MHz) reactive sputter deposition was carried out (see Table 1 for details) after reaching a base pressure of about $2 \times 10^{-4} \mathrm{~Pa}$. The target consisted of a hyperpure silicon wafer ${ }^{6}$ spaced about $50 \mathrm{~mm}$ from the substrates. It should be noted that a $70 \mathrm{~mm}$ diameter 'unbalanced' magnetron was used (with track radius of about 40-50 $\mathrm{mm}$ ) for the deposition experiments. The negative 'self bias' of the target was high and ranged from $650 \mathrm{~V}$ to $950 \mathrm{~V}$ dc depending, of course, on sputter conditions. During sputtering at low sputter gas pressures $(\mathrm{p} \approx 0.5 \mathrm{~Pa}$ ), a negative self bias of the insulated sample holder of about $-20 \mathrm{~V}$ (relative to ground level) developed, which enhanced the ion bombardment of the growing film. At higher sputter gas pressures $(p>1 \mathrm{~Pa})$, the self bias of the samples levelled at about $-4 \mathrm{~V}$, which corresponds to the plasma potential. All thin films contained about $2 \%$ of copper, introduced by placing small copper pins into the main erosion area of the magnetron.

After taking the samples out of the machine, compositional analysis was done by ERD/RBS measurements and the film thickness and the refractive index were determined from the optical transmittance and reflectance. Raman scattering provided information about the crystalline structure and X-ray diffraction measurements provided the thin film orientation and grain size. Scanning electron microscopy (SEM) completed the analysis. 
During the Elastic Recoil Detection (ERD) experiments, a $2 \mathrm{MeV}$ He beam was directed onto the samples tilted at an angle of $75^{\circ}$. The recoiled hydrogen ions were collected by a detector situated at an angle of about $30^{\circ}$ relative to the incident beam. The detector was protected by a $9 \mu \mathrm{m}$ thick Kapton foil to stop scattered He ions. A second detector placed at $160^{\circ}$ measured the backscattered He ions ${ }^{7}$. At this angle of incidence and in the thickest samples, the incident He beam did not reach the substrate and the measurements yielded the ratio between hydrogen and silicon in about a $300 \mathrm{~nm}$ thick zone at the sample surface. RBS experiments at normal incidence allowed a measurement of the absolute number of silicon atoms present in the thin film.

From the transmittance and reflectance of the samples in the optical and near infrared (wavelengths $\lambda$ between 600 and $1500 \mathrm{~nm}$ ), the film thickness and refractive index were estimated. The results were analysed according to a procedure proposed by Minkov ${ }^{8}$. This technique estimates the film thickness with an error less than 5\%. Comparing the thickness with the absolute number of atoms present in the thin film, as determined by the RBS measurements, the film density $\rho$ was readily estimated.

The Raman scattering equipment (Jobin Ivan ${ }^{9}$ ) consisted of a cw-Argon laser (power about $5 \mathrm{~mW}$ (2.3 $\mathrm{eV}$ ) at normal incidence), a triple monochromator and a detection system with multichannel plate, CCD camera and associated hard- and software. The resolution of the system is about $2.0 \mathrm{~cm}^{-1}$ and we averaged consistently over three scans. All experiments were done at room temperature. The background originating from the glass substrates was eliminated by a careful data treatment taking into account beam attenuation and related effects. The diameter of the analysed area was about $\Phi=50 \mu \mathrm{m}$. X-ray diffraction spectra were recorded by a Philips Geiger counter diffractometer (PW 1719) using $\mathrm{CuK} \alpha$ radiation. Due to the weak interaction between X-rays and $\mathrm{Si}$ atoms within the thin films, the signal to noise ratio was always relatively low and could not be increased significantly by increasing the measuring time.

\section{Results and discussion}

\subsection{Composition}

The RF reactive magnetron sputter experiments produced hydrogenated $\mu \mathrm{c}-\mathrm{Si}$ thin films with an absolute hydrogen content of about 27-33 at.\%. Although we could not find a simple relationship between the deposition parameters and the hydrogen content, the latter tends to increase with decreasing argon sputter gas pressure and with decreasing substrate temperature. The hydrogen 
originates mainly from $\mathrm{Si}-\mathrm{H}$, bonds as verified by FTIR measurements ${ }^{10}$. The oxygen content within most of the thin films was below 3 at.\%, which corresponds to the detection level at the experimental conditions during RBS measurements. Therefore, a possible contribution of hydrogen from water, captured in pores within the thin film could also be estimated to be lower than about $3 \%$. Nevertheless, thin films deposited at the lowest deposition rates (RF sputter power 40-80 W) showed an increased oxygen content (up to 12 at.\%) and the $\mu \mathrm{c}-\mathrm{Si}: \mathrm{H}$ film deposited during experiment a4 at a somewhat higher background pressure, contained about 18 at.\% oxygen. These films also reveal an increased hydrogen content indicating the presence of $\mathrm{OH}$-groups. For low deposition rates, the oxygen content can be reduced significantly by improving the base pressure of the sputter equipment.

\subsection{Microstructure of thin films produced by RF sputtering}

Thin films deposited by sputter deposition processes show, as is generally observed, a columnar structure. The column diameter ranges from 50 to $150 \mathrm{~nm}$, see Figures 1 (a) and (b), within films with a thickness from $0.3 \mu \mathrm{m}$ to $1 \mu \mathrm{m}$. The columns themselves consist of small (nano) crystals, and the size of these crystals can be influenced within a wide range by the deposition parameters. The X-ray diffraction analysis of all samples reveals that all hydrogenated silicon films have a crystalline component. Figure 2 shows a typical X-ray rocking curve between $20^{\circ}$ and $55^{\circ}$. The crystals are small, with a size up to $30 \mathrm{~nm}$ (as determined by application of the Scherrer equation) ${ }^{11}$ and show a preferred orientation with the (111) plane parallel to the substrate surface. Only a few samples show a weak peak corresponding to the (220) plane. Due to the low intensity and the preferred film orientation, we could not study systematically the peak width to distinguish between peak broadening due to a variation of the grain size or due to other effects like intrinsic stress, structural defects, etc. 


\begin{tabular}{|c|c|c|c|c|c|c|}
\hline Experiment & $p_{\mathrm{H}_{3}}(\mathrm{~Pa})$ & $p_{\text {atr }}(\mathrm{Pa})$ & $\begin{array}{l}\text { Temperature } \\
(\mathrm{C})\end{array}$ & $\begin{array}{l}\text { RF power } \\
\text { (W) }\end{array}$ & $\begin{array}{l}\text { Deposition-rate* } \\
\left(\mathrm{nm} \mathrm{s}^{-1}\right)\end{array}$ & $\begin{array}{l}\text { Si deposition-rate } \dagger \\
\left(\text { nm s }^{-1}\right)\end{array}$ \\
\hline al & 0.15 & 0.33 & 250 & 40 & 0.023 & 0.014 \\
\hline $\mathrm{a} 2$ & 0.10 & 2.0 & 250 & 40 & - & 0.018 \\
\hline a 3 & 0.27 & 0.33 & 220 & 80 & - & 0.013 \\
\hline $\mathrm{a} 4$ & 0.27 & 0.38 & 220 & 80 & - & 0.027 \\
\hline a5 & 0.15 & 0.33 & 250 & 100 & 0.14 & 0.063 \\
\hline 36 & 0.27 & 5.0 & 250 & 180 & 0.27 & 0.13 \\
\hline a7 & 0.15 & 2.0 & 250 & 200 & 0.49 & 0.20 \\
\hline a8 & 0.04 & 0.60 & 250 & 300 & 0.86 & 0.48 \\
\hline a 9 & 0.07 & 0.70 & 250 & 300 & 0.66 & 0.47 \\
\hline 410 & 0.10 & 2.0 & 250 & 300 & 0.56 & 0.31 \\
\hline al1 & 0.15 & 2.0 & 200 & 300 & 0.65 & 0.39 \\
\hline a 12 & 0.15 & 20 & 250 & 300 & 0.80 & 0.33 \\
\hline a 13 & 0.15 & 2.0 & 300 & 300 & 0.57 & 0.44 \\
\hline a 14 & 0.15 & 2.0 & 250 & 400 & 0.93 & 0.43 \\
\hline a 15 & 0,07 & 0.45 & 200 & 500 & 1.08 & 0.63 \\
\hline
\end{tabular}
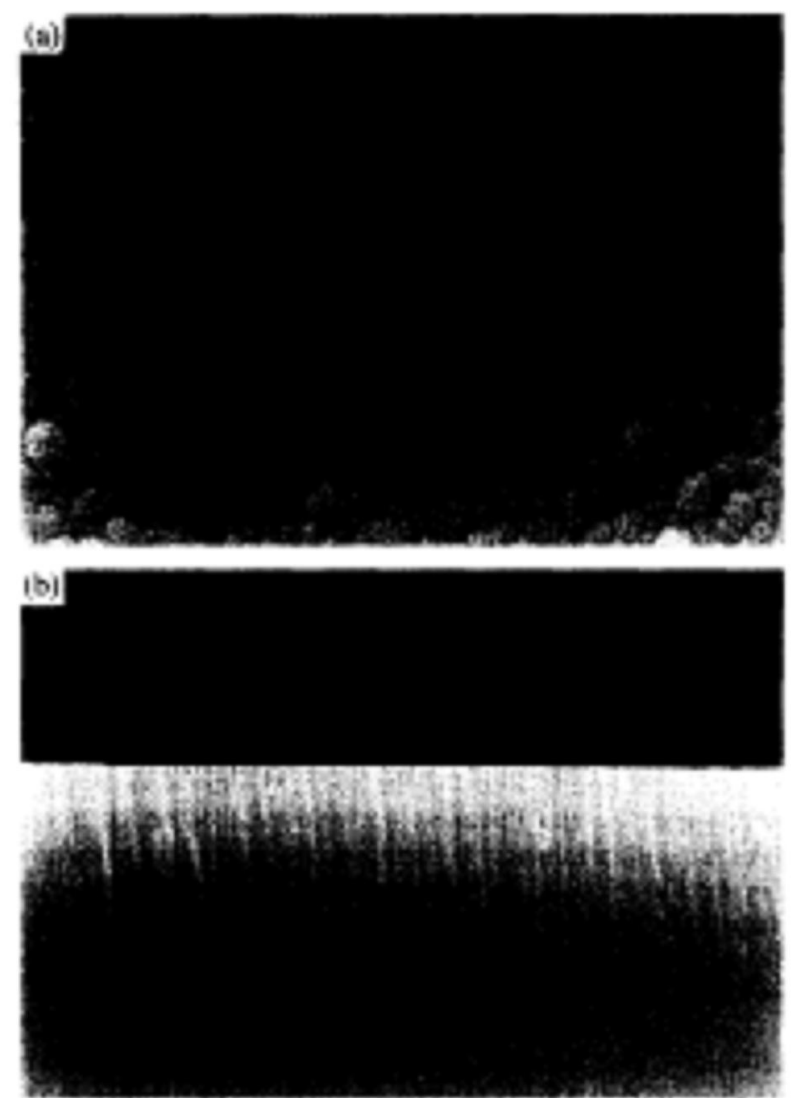

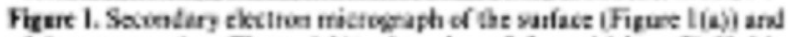

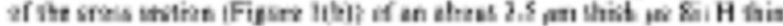
Shn icopcritsth a 16 . 
Raman spectroscopy, being a very sensitive technique, detects details of the microstructure. The Stokes peak in the vicinity of $520 \mathrm{~cm}^{-1}$ is generally attributed to the transverse optical mode (TO) of crystalline $\mathrm{Si}$. This peak is shifted to smaller wavenumbers for decreasing crystal diameter D within the substrate plane. The deconvolution of a typical Raman spectrum, see Figure 3, reveals three Lorentzian shaped peaks : one between 495 and $521 \mathrm{~cm}^{-1}$ (component $\mathrm{Ll}$ of the Raman spectrum in Figure 3) which is related to the crystal diameter D, a broad peak at about $480 \mathrm{~cm}^{-1}$ which is related to the amorphous phase ${ }^{12}$ (component L2 in Figure 3) and a relatively important peak at $490 \pm 2 \mathrm{~cm}^{-1}$ (component L3 in Figure 3), which is frequently found in Si thin films with very small crystals ${ }^{12}$ and can also be attributed to vibrations in the transverse optical mode. During the deconvolution of Raman spectra the positions of components L2 and L3 were fixed at $480 \mathrm{~cm}^{-1}$ and $490 \mathrm{~cm}^{-1}$, respectively.

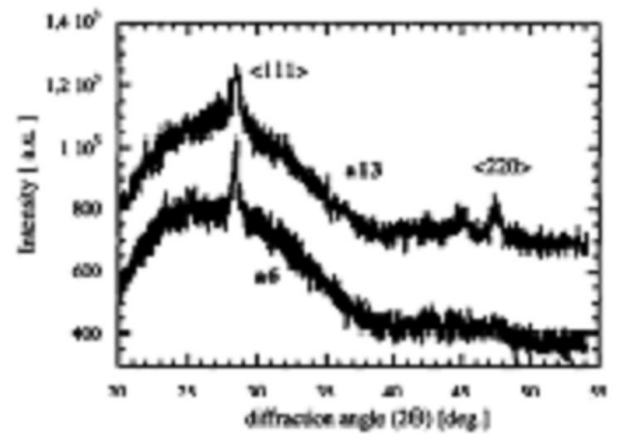

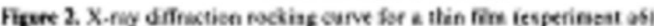

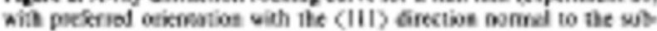

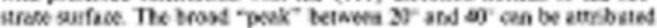
nuhty to the eter wherratt

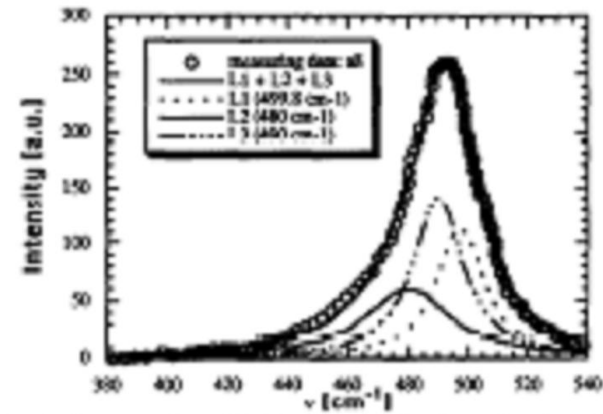

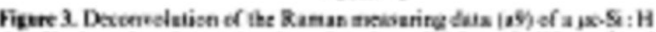

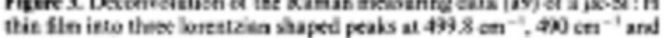

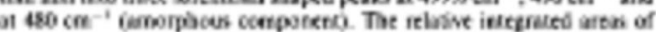

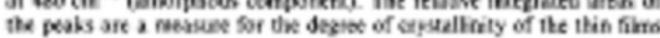
the with.

Based on theoretical considerations and experimental findings ${ }^{12,13}$, we calculated the crystal size of all samples from the position of the $\mathrm{Ll}$ peak, as it is represented in Table 2. To simplify the calculations, we acknowledged a linear relation between grain size and peak position in the range from $490 \mathrm{~cm}^{-1}$ (D $\approx 0.3 \mathrm{~nm})$ to $516 \mathrm{~cm}^{-1}(\mathrm{D}=6.5 \mathrm{~nm})$. Of course, the deconvolution of the Raman spectra and the subsequent determination of the grain size does not account for a possible grain size distribution. 


\begin{tabular}{|c|c|c|c|c|c|c|}
\hline Experiment & $\begin{array}{l}\text { Position L. } \\
\left(\mathrm{cm}^{-1}\right)\end{array}$ & $\begin{array}{l}D \\
(\mathrm{~mm})\end{array}$ & c. & 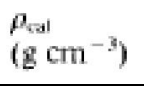 & $\begin{array}{l}\rho_{\text {eqp }} \\
\left(\mathrm{g} \mathrm{cm}^{-1}\right)\end{array}$ & $\begin{array}{l}\text { Hydrogen } \\
\text { content (at. \%) }\end{array}$ \\
\hline al & 517.1 & 6.8 & 0.86 & 2.24 & 2.19 & 50 \\
\hline 22 & 510.6 & 52 & 0.78 & 2.21 & & 50 \\
\hline a 3 & 516.5 & 6.6 & 0.82 & 2.21 & & 32 \\
\hline $\mathrm{a} 4$ & 521.0 & 12.0 & 0.68 & 2.13 & & 46 \\
\hline a 5 & 5210 & 12.0 & 0.23 & 1.85 & 1.63 & 27 \\
\hline$a 6$ & 504.4 & 3.6 & 0.38 & 1.94 & 1.65 & 26 \\
\hline 7 & 506.5 & 4.1 & 0.13 & 1.78 & 1.47 & 32 \\
\hline a 8 & 499.8 & 2.5 & 0.79 & 2.20 & 2.00 & 38.6 \\
\hline a 9 & 509.8 & 5.0 & 0.55 & 2.04 & 2.52 & 28.6 \\
\hline a 10 & 497.5 & 1.9 & 0.32 & 1.90 & 1.97 & 27 \\
\hline all & 496.6 & 1.6 & 0.29 & 1.88 & 2.16 & 33 \\
\hline a 12 & 495.7 & 1,4 & 0.57 & 2.06 & 1.45 & 28.6 \\
\hline a 13 & 500.4 & 2.6 & 0.27 & 1.87 & & 28.5 \\
\hline a 14 & 499.0 & 2.2 & 0.18 & 1.81 & 1.72 & - \\
\hline a 15 & 495.3 & 1.3 & 0.77 & 2.19 & 2.09 & 44.4 \\
\hline
\end{tabular}

The relative, integrated intensity of the three Raman peaks is related to the relative amount $\mathrm{c}$ of the crystalline fraction within the thin film. Equation 1 shows this relation taking into account the crystal size dependent scattering cross section ${ }^{14}$

$$
c=\frac{I_{\mathrm{c}}}{I_{\mathrm{c}}+I_{2}(0.1+\exp (-D / 25 \mathrm{~nm}))}
$$

$I_{c}$, and $I_{a}$, represent the integrated intensity of the crystalline (Ll) and amorphous (L2) Raman peaks, respectively and D the crystal diameter. It should be noted, that the decay length of the exciting laser beam (about $25 \mathrm{~nm}$ ) was estimated based on the assumption of spherical micro-crystals in an amorphous matrix.

Table 2 shows the crystalline fraction $\mathrm{c}$ of the thin $\mu \mathrm{c}-\mathrm{Si}: \mathrm{H}$ thin films. Comparing the grain size estimated from the Raman scattering experiments (TO mode) and X-ray diffraction (Scherrer formula), we tend to expect Si crystals of non-spherical shape, with a lateral extension as indicated by the Raman experiments. The grain boundaries between the nano crystals occupy a relatively large volume fraction. It is this volume fraction which can be attributed to the 'amorphous' phase within the $\mu \mathrm{c}-\mathrm{Si}: \mathrm{H}$ thin films, e.g. assuming a columnar crystal with diameter $\mathrm{D} \approx 2.6 \mathrm{~nm}$ in sample a8, the grain boundary will occupy more than $30 \%$ of the total volume of the column, as illustrated in Figure 4 . Generally speaking, a small grain size will be accompanied by a low degree of crystallinity of the thin film ${ }^{15}$ (as is also indicated in Figure 5). 


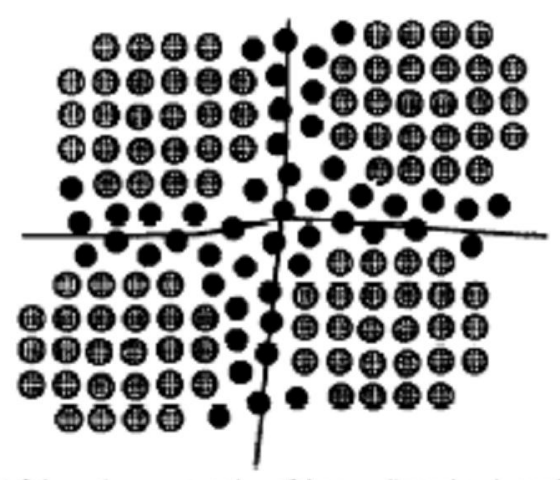

Figure 4. Sehematic representation of the two-dimensional atomic strueture of $u_{-\mathrm{e}} \mathrm{Si}$ : $\mathrm{H}$ thin films. The atoms in the centre of the crystals are indicated by the shaded circles. The atoms in the boundary core regions are represented in black. The boundary regions are characterised by a reduced density.

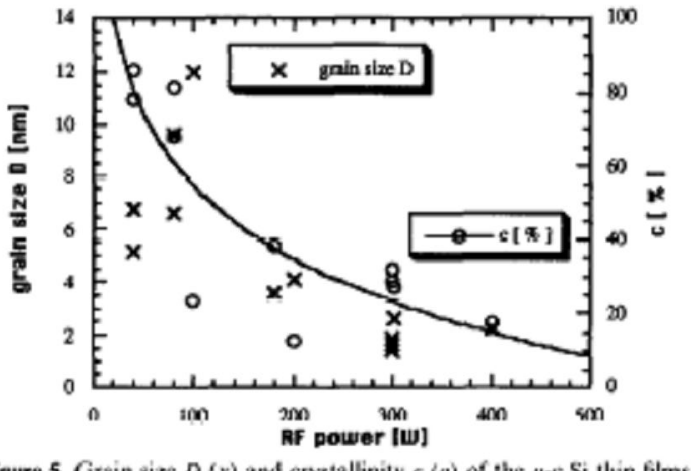

Fisure 5. Grain size $D(x)$ and crystallinity $c(v)$ of the $\mu-\mathrm{c}$ Si thin films produced at a sputter gass pressure of ahout $2 \mathrm{~Pa}$ as a function of the RF power $P[W]$ (the solid bine in the figure was introduced solely to guide the eye).

The thin film density $\rho_{\text {cal }}$, can be computed by the relative contribution of crystalline component $\mathrm{c}$ of the $\mu$-Si-H thin films according to equation (2), assuming a density $\rho_{\mathrm{c}}$ of $2.33 \mathrm{~g} \mathrm{~cm}^{-3}$ and $\rho_{\mathrm{a}}=1.7 \mathrm{~g} \mathrm{~cm}^{-}$ ${ }^{316}$ for the two components, respectively.

$$
\rho_{\text {sal }}=2.33 c+1.7(1.0-c)
$$

Nevertheless, other effects, typical for low temperature sputter deposited thin films like voids between the growing columns, structural defects and the hydrogen content will also decrease the film density. The measured density $\rho_{\text {exp }}$, as determined from the number of Si and $\mathrm{H}$ atoms in the $\mu \mathrm{c}-\mathrm{Si}: \mathrm{H}$ film (RBS-measurements) and the film thickness (measurement of optical transmittance and reflectance) is therefore lower than the calculated density. On average, the measured density is about $10 \%$ lower than the calculated film density, indicating a void volume of about $10 \%$. The void volume also depends somewhat on the sputter conditions as presented in Figure 6.

The $\mu \mathrm{c}-\mathrm{Si}: \mathrm{H}$ films were remarkably stable. A $5 \mathrm{~h}$ annealing in vacuum at $500^{\circ} \mathrm{C}$ does not influence significantly the microstructure of the $\mu \mathrm{c}-\mathrm{Si}: \mathrm{H}$, neither does it cause any damage in the thin film, as is reported by Ruther ${ }^{17}$.

\subsection{Relationship between microstructure and sputter parameters}

The microstructure of thin films produced by sputter techniques is generally a function of intrinsic deposition parameters such as (i) sputter particle energy, (ii) substrate temperature and (iii) deposition rate $^{18}$. The sputtered particle energy, when incident on the growing film, depends strongly on the sputter gas density (gas pressure and temperature) and target-substrate distance ${ }^{19}$. Increasing the mean sputtered particle energy will increase, due to collisional process within the growing film ${ }^{20}$, the mean 
diffusion length $\Lambda$ of the deposited particles. Of course, thermal diffusion within the deposited film, along grain boundaries and on the surface will also take place. The surface diffusion, which is a relatively fast process, is hindered by a high arrival rate (deposition rate) of $\mathrm{Si}$ atoms from the target. We will not consider other effects like neutral particle bombardment and ionisation rate of the deposited Si particles, which will also influence the mean diffusion length $\Lambda$. Nevertheless, it should be mentioned that in our experimental configuration $\Lambda$ is enhanced by the so-called 'unbalanced' magnetron. We will discuss now the influence of the three above mentioned parameters on the crystallinity of the growing $\mu \mathrm{c}-\mathrm{Si}: \mathrm{H}$ thin film.

Increasing the sputter power will increase the deposition rate and therefore diminish the time available for the deposited particles to diffuse via (surface) diffusion processes. The diminished diffusion length $\Lambda$ of the deposited particles hinders the grain growth within the produced thin film as shown in Figure 5 and therefore also reduces the crystalline fraction $\mathrm{c}$ of the $\mu \mathrm{c}-\mathrm{Si}: \mathrm{H}$ thin film.

The diminished diffusion length due to an enhanced deposition rate also causes an increased thin film porosity. A comparison between the number of $\mathrm{Si}$ and $\mathrm{H}$ atoms present in the thin film, as determined by RBS/ERD measurements, and the actual film thickness, as determined from optical transmittance and reflectance, as a function of the sputter power reveals an increasing gap. This gap, as shown in Figure 6, indicates an increasing film porosity with increasing deposition rate. (To simplify the comparison we present the deposition rate computed from final film thickness and final mass (RBS measurements), respectively in Figure 6.) Augmenting the sputter gas pressure at constant targetsubstrate distance and constant power decreases the mean sputtered particle energy and therefore the collisional processes within the growing film. Figure 7 shows the relation between the sputter gas pressure and the mean grain size indicating an increased crystallinity with enhanced mobility of the arriving particles. Of course, the stopping power of the hydrogen component of the sputtering gas for silicon atoms is much lower than the stopping power of the argon component. Therefore, the argon partial pressure is used in Figure 7. 


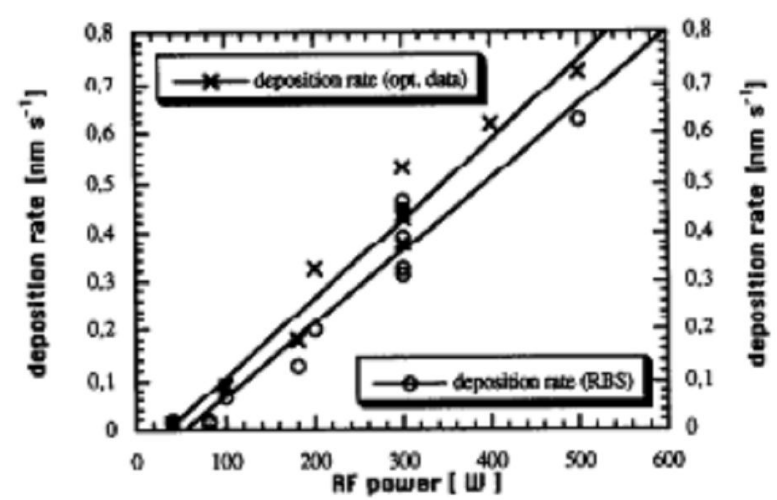

Figure 6. Deposition rate of $\mu \mathrm{c}-\mathrm{Si}: \mathrm{H}$ as a function of the RF sputter power. The deposition rate was calculated from the final fila thickues as measured from the optical transmittance. The deposition rate of $\mathrm{Si}$ and $H$ was measured by RBS/ERD and the deposition rate than computed assuming a thin film density of $2.33 \mathrm{~g} \mathrm{~cm}^{-5}$.

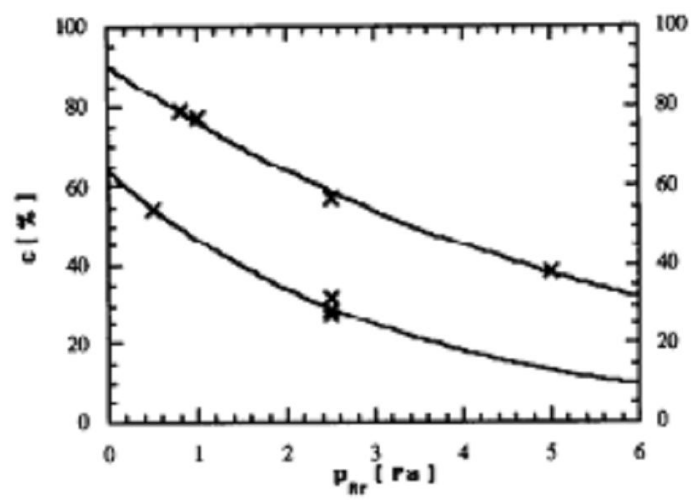

Figure 7. Crystallinity of the rec- $\mathrm{Si}$ : H thin films produced at at RF power of abuut $300 \mathrm{~W}$ as a function of the spatter gas pecssure $\rho_{\text {Ar }}$ (the solid lines in the figure were introduced to indicate the scatter of the data due to sputter gas pressure and substrate temperature variations).

The substrate temperature $\left(\mathrm{T}_{\mathrm{s}}\right)$ was varied between $473 \mathrm{~K}$ and $573 \mathrm{~K}$ during the deposition, but we could not find any significant influence of this temperature on grain size. The ratio between the substrate temperature and the $\mathrm{Si}$ melting temperature $\mathrm{T}_{\mathrm{M}}=1685 \mathrm{~K}$, is in some way a measure for the diffusion length. Since in our experiments the temperatures were much lower than $T_{M}$ and the range of variation was only about $20 \%$, it is not surprising that the grain size did not depend on $\mathrm{T}_{\mathrm{s}}$. It should be noted again, that even annealing the thin films for $5 \mathrm{~h}$ at $500^{\circ} \mathrm{C}$ in vacuum does not increase the grain size, significantly.

\section{Conclusions}

In light of this work on the microstructure of thin films of $\mu \mathrm{cSi}: \mathrm{H}$ produced by RF planar magnetron sputtering, the experimental results can be summarised as follows : $\mu \mathrm{c}-\mathrm{Si}: \mathrm{H}$ thin films can be produced by RF planar magnetron sputtering with a grain diameter up to $100 \mathrm{~A}$, at reasonable deposition rates. These grains are of non-spherical shape with the bigger dimension normal to the substrate surface. The crystals are generally oriented with the (111) direction normal to the substrate surface.

The thin film microstructure is dominated by Si nano crystals and an 'amorphous' component which can be attributed to the grain boundaries. These crystals form the columnar structure of the thin film.

Most significant for the grain size is the deposition rate, which depends of course on sputter power density. Low deposition rates favour large grains; other deposition parameters such as sputter gas pressure and substrate temperature are less significant for the grain growth.

The hydrogen content of the thin films is about 27-33 at.\% and no simple relation to sputter conditions, crystallinity or grain size could be found. 


\section{Acknowledgements}

We gratefully acknowledge the supply of the hyperpure silicon targets by Dr Hoffmann from Wacker, Burkhausen, Germany and the X-ray diffraction measurements by Mr Azevedo, Universidade do Minho.

\section{References}

${ }^{1}$ L Yang, L Chen, S Wiedeman and A Catalano, Mat Res Soc. Symp. Proc, 283,463 (1993).

${ }^{2}$ R C Ross and R Messier, J Appl Phys, 52(8), 5329 (1981).

${ }^{3}$ M Komori, S Furukawa and T Miyasato, Phys Letters A, 135, 401 (1989).

${ }^{4}$ Z Iqbal, S Vepfek and A P Webb, Appl Phys Lett, 36, 163 (1980).

${ }^{5}$ T Imura, et al. Jap J Appl Phys, 23, 179 (1984).

${ }^{6}$ Wacker-Chemitronic GmbH, Johannes-Hess-Str 24, D-84489 Burkhausen, Germany.

${ }^{7}$ F Paszti, E Kotai, G Mezey, A Manuaba, L Pocs, D Hildbrandt and H Strusny, Nucl Instr and Meth. B15,486 (1986).

${ }^{8}$ DA Minkov, J Phys D, 22, 199 (1989).

${ }^{9}$ Jobin Ivon, 1618, rue du canal, F-91 165 Longjumeau Cedex, France.

${ }^{10}$ to be published

${ }^{11}$ V H Tiensuu, S Ergun, L E Alexander, J Appl Phys., 35, I718 (1964).

${ }^{12}$ G Kanellis, J F Morhange and M Balkanski, Phys Rev. B, 21, 1543 (1980).

${ }^{13}$ Z Iqbal S Vepfek, A P Webb and P Capezzuto, Solid State Communications, 37,993 (1981).

${ }^{14}$ E Bustarret, M A Hachicha and M Brunel, Appl Phys Lett, 52, 1675 (1988).

${ }^{15}$ H Gleiter, Nano Structured Materials, 1,1 (1992).

${ }^{16}$ G Mvburu. R Swaneuoel. Thin Solid FilmA. 149. 331 (1987).

${ }^{17}$ R Rither:J Livingstone, Thin Solid Films, 251,30 (1994).

${ }^{18}$ e.g. J E Greene, in Multicomponent and Multilayered Thin Films for Advenced Microtechnoloyies : Techniques, Fundamentals and Devices. NATO-AS1 series E 234. p 39 (1992).

${ }^{19}$ A Gras-Marti and J A Valles-Abarca, J Appl Phys, 54, 1071 (1983).

${ }^{20}$ C M Gilmore and J A Sprague, Phys. Rev. B 44,895O (1991). 